University of Nebraska - Lincoln

DigitalCommons@University of Nebraska - Lincoln

October 1995

Die Registrierung der Orgelwerke J. S. Bachs

Quentin Faulkner

University of Nebraska - Lincoln, qfaulkner1@unl.edu

Follow this and additional works at: https://digitalcommons.unl.edu/musicfacpub

Part of the Music Commons

Faulkner, Quentin, "Die Registrierung der Orgelwerke J. S. Bachs" (1995). Faculty Publications: School of Music. 21.

https://digitalcommons.unl.edu/musicfacpub/21

This Article is brought to you for free and open access by the Music, School of at DigitalCommons@University of Nebraska - Lincoln. It has been accepted for inclusion in Faculty Publications: School of Music by an authorized administrator of DigitalCommons@University of Nebraska - Lincoln. 


\title{
Die Registrierung der Orgelwerke J. S. Bachs
}

\author{
Von Quentin Faulkner (Lincoln, Nebraska)
}

„Es versteht sich übrigens von selbst, daß beim Gebrauch die gewählten Verbindungen von Stimmen mit dem Geist und Sinn der vorzutragenden Stücke harmonieren müssen, sonst ist das Ganze nur eine theoretische Spielerei, ohne allen künstlerischen Wert.“1

So formulierte Friedrich Konrad Griepenkerl, der erste Herausgeber von Bachs Orgelwerken, zusammenfassend die Bedeutung der angemessenen Registerwahl für das Spiel dieser Kompositionen. Ein erfahrener Organist liest diese Worte jedoch nicht ohne gewisse Bedenken. Wer hilft einem bei der Wahl der passenden Registrierungen? Die verschiedenen Vorschläge Griepenkerls vergrößern eher die Unsicherheit des Organisten; sie sind zwar sinnvoll, aber zu allgemein, um praktischen Wert zu haben. Auch stellt sich die Frage nach ihrer Authentizität. Weiterhin ist zu klären, auf welche Voraussetzungen sich Griepenkerls Bemerkungen stützen und inwieweit er Vorstellungen von Bach selbst beziehungsweise von dessen Schülern vertritt.

Die beiden letzteren Fragen stellen sich Organisten erst in jüngerer Zeit. Zweifellos bezogen sich die Registrierungsvorschläge Griepenkerls auf Orgeln seiner eigenen Epoche, nicht aber auf die der Bach-Zeit. Das gleiche gilt mutatis mutandis auch für die Anleitungen von Albert Schweitzer ${ }^{2}$ oder von Hermann Keller $^{3}$. Erst mit dem neuerwachten Interesse an den Eigenschaften einer idealen „Bach-Orgel“" und mit dem Versuch, deren Prinzipien zu verstehen und klanglich $\mathrm{zu}$ verwirklichen, interessieren sich Organisten auch dafür, wie man die Orgelwerke auf verschiedenen Orgeltypen stilgerecht registrieren kann, vor allem aber, wie Bach selbst es gehalten haben könnte und wie er seine Schüler instruiert haben mag.

Dieses Interesse wird jedoch angesichts der spärlichen Quellen aus dem direkten Umfeld Bachs enttäuscht. Immerhin gibt es eine Abhandlung aus der Feder eines Bach-Schülers, die Ausführungen über Orgelregistrierung enthält und die einen gesicherten Ausgangspunkt für die Ergänzung und Klärung anderer bereits bekannter Quellen aus dem Bach-Kreis bietet. Überdies erlaubt sie, genauer als bisher andere zeitgenössische Quellen zur Orgelregistrierung hinsichtlich ihrer Zuständigkeit für Bachs Praxis zu überprüfen.

Die Abhandlung findet sich im dritten Band von Friedrich Wilhelm Marpurgs Periodikum Historisch-Kritische Beyträge zur Aufnahme der Musik. ${ }^{4}$ Auf den ersten Blick handelt es sich hierbei um die Rezension einer 1757 in Breslau

${ }^{1}$ Vorrede zu: J. S. Bach's Kompositionen für die Orgel, Band I, Leipzig 1844 (1. Auflage), S. II.

2 Ch.-M. Widor und A. Schweitzer, Johann Sebastian Bach: Complete Organ Works, New York 1912.

${ }^{3}$ H. Keller, Die Orgelwerke Bachs, Leipzig 1948, S. 32-38.

${ }^{4}$ Berlin 1758, Sechstes Stück, S. 486-518. 
erschienenen Sammlung von Orgeldispositionen, ${ }^{5}$ in Wirklichkeit befaßt sich jedoch nur der einleitende Abschnitt mit der neuen Publikation. Dieser Umstand hat zusammen mit der Fehlzuschreibung an Marpurg eine Reihe von Wissenschaftlern irregeleitet und die Bedeutung des Essays bisher verschleiert. Die Unsicherheit bezüglich der Autorschaft ist verständlich, da weder das Inhaltsverzeichnis des sechsten Stücks noch der Titel der Abhandlung den Namen des Verfassers nennen. Einzig der Name „Agricola“ am Ende des Essays identifiziert diesen als Johann Friedrich Agricola (1720-1774), Bachs Schüler von etwa 1738 bis 1741 . Trotz einer erfolgreichen musikalischen Laufbahn (Friedrich der Große ernannte ihn 1751 zum Königlich Preußischen Hofkomponisten) hatte Agricola nie ein wichtiges Organistenamt inne. ${ }^{6}$ Inwiefern sollten also seine Vorschläge zur Orgelregistrierung von Belang sein? Und mit welcher Begründung wäre anzunehmen, daß sie die Praxis seines Lehrmeisters reflektieren? Eine Beantwortung dieser Fragen hätte sowohl Agricolas Stellung innerhalb des Bach-Kreises als auch Zeugnisse seiner Wertschätzung von Bachs musikalischem Vermächtnis zu berücksichtigen.

Agricola wirkte von 1741 bis 1774 in Berlin, Carl Philipp Emanuel Bach ebenda von 1738 bis 1768 sowie Johann Philipp Kirnberger von etwa 1751 bis 1783 . Dieses „Berliner Triumvirat“ ehemaliger Bach-Schüler bildete das Zentrum der Bach-Pflege in Berlin, nach Bachs Tod wurde es sogar zum Mittelpunkt des gesamten Bach-Kreises. Zusammen mit C. P. E. Bach verfaßte Agricola den 1754 veröffentlichten Nekrolog auf Johann Sebastian Bach. ${ }^{7}$ Im Jahre 1769 arbeitete er mit dem Verleger Friedrich Wilhelm Birnstiel an der Herausgabe des zweiten Bandes der frühesten gedruckten Sammlung von Bachs vierstimmigen Chorälen (Band 1 wurde 1765 von Friedrich Wilhelm Marpurg und C. P. E. Bach herausgegeben). Zwischen diese beiden Daten fällt die Veröffentlichung der Abhandlung in Marpurgs Periodikum. Den Namen Johann Sebastian Bach erwähnt Agricola in seinem Essay kein einziges Mal. ${ }^{8}$ Doch seine ständigen Bemühungen um die Verbreitung von Bachs Musik wie auch sein enger Kontakt zu anderen Schülern und Verehrern des Meisters vermitteln einen Eindruck davon, in welchem Maße der große Respekt vor den Intentionen seines Lehrers Agricolas Denken geprägt haben muß.

Parallel zu dessen Mitarbeit bei der Herausgabe der Bach-Choräle betraute Birnstiel Agricola mit der Schlußredaktion von Jacob Adlungs hinterlassenem Orgellehrbuch Musica mechanica organoedi, das 1768 im Druck herauskam. Auch Adlungs ursprünglicher Text erwähnt Johann Sebastian Bach nirgends. Agricolas relativierende Kommentare zu bestimmten Auffassungen Adlungs

${ }^{5}$ Sammlung einiger Nachrichten von berühmten Orgel=Wercken in Teutschland, verlegts Carl Gottfried Meyer. Meyer könnte auch der Herausgeber der Sammlung sein.

${ }^{6}$ Ch. Burney schreibt allerdings: „Man hält ihn [Agricola] für den besten Orgelspieler in Berlin ..." (Carl Burney's der Musik Doctors Tagebuch seiner Musikalischen Reisen, Bd. 3, Hamburg 1773; Faks.-Ausgabe, hrsg. von R. Schaal, Kassel 1959), S. 59.

${ }^{7}$ Dok III, Nr. 666.

${ }^{8}$ Ich stelle weiter unten jedoch die Überlegung an, daß zumindest eine von Agricolas Bemerkungen sich auf eine selbsterlebte Darbietung Bachs auf der Orgel bezieht. 
berufen sich jedoch dreimal auf Bach $^{9}$ als offensichtlich größere Autorität. In seinem Essay von 1758 schildert Agricola verständlicherweise seine eigenen Ansichten zur Orgelregistrierung. Es ist allerdings mehr als wahrscheinlich, daß einige seiner Standpunkte denjenigen seines Lehrers sehr nahekommen. Möglicherweise ist hierbei nur ein späteres Stadium von Bachs Überlegungen zur Registrierung festgehalten. Da dieser jedoch in solchen Dingen vorausschauend zu denken pflegte, ließe sich annehmen, daß er viele seiner Überzeugungen schon in seinen früheren Jahren vertreten hat.

\section{QUELLEN DES FRÜHEN 18. JAHRHUNDERTS ZU FRAGEN DER REGISTRIERUNG}

Agricolas Essay von 1758 ist bekanntlich nicht die einzige Quelle zur mitteldeutschen Registrierpraxis der ersten Hälfte des 18. Jahrhunderts. Die greifbaren Zeugnisse lassen sich lose in drei Gruppen einteilen. Zumindest eine Quelle aus jeder dieser Gruppen weist eine Verbindung zum Bach-Kreis auf.

Gruppe 1: Abhandlungen über allgemeine Prinzipien der Registrierung oder Kommentare zu spezifischen Fragen (mit knappen Registrieranweisungen, jedoch ohne Bezug auf bestimmte Werke oder Werkgruppen).

In seinem Vollkommenen Capell-Meister ${ }^{10}$ erläutert Johann Mattheson seine Ansichten über die Zusammensetzung des Plenums:

„Es gehören zum vollen Werck die Principale, die Sordunen, die Salcionale oder Salicete (Weiden-Pfeiffen) die Rausch-Pfeiffen, die Octaven, die Qvinten, Mixturen, Scharffen (kleine Mixturen von drey Pfeiffen) Qvintadeen, Zimbeln, Nasat, die Terzien, Sesqvialtern, Super-Octaven, Posaunen im Pedal, nicht im Manual: denn die Posaunen sind ein Rohr-Werck, welches aus dem Manual, bey voller Orgel, ausgeschlossen bleibet; indem es daselbst, wegen der Höhe, zu sehr schnarren würde; da es hergegen, wegen der Tiefe des Klanges, im Pedal prächtig lautet, wenn die Mundstücke, wie billig, gefüttert sind.“

Obwohl Mattheson in Norddeutschland tätig war, gleichen seine Erläuterungen zum Plenum denen der folgenden, in Mitteldeutschland beheimateten Quelle.

Jacob Adlung widmet das gesamte achte Kapitel seiner Musica mechanica organoedi dem Gebrauch der Register. Obwohl sein Traktat erst 1768 veröffentlicht wurde, scheint er im wesentlichen bereits in den 1720er und 1730er Jahren entstanden zu sein. Jedenfalls repräsentiert diese Schrift die Ansichten eines jüngeren Zeitgenossen Bachs (Adlung lebte von 1699 bis 1762). Die Anweisungen für die Zusammenstellung des Plenums ( $\$ 231$ und $\S 234)$ ähneln denjenigen bei Mattheson, doch verlangt Adlung ausdrücklich möglichst mehrere zusätzliche 16'-Register im Manual. Adlungs Methode der Registerwahl für Kombinationen außerhalb des Plenums postuliert eine Reihe von Grundregeln, ${ }^{11}$ in deren Rahmen sodann alle möglichen Permutationen von Registerkombinationen ausgeschöpft werden; diese Methode

9 Band 1, S. 66 und 187; Band 2, S. 23-24. Vgl. Dok III, Nr. 739 und 742.

${ }^{10}$ Hamburg 1739, S. 467, § 76.

11 So müssen Äqualregister stets die tiefste Lage einnehmen und vor Aliquotstimmen den Vorrang haben. 
deutet auf das Fehlen jeglicher kodifizierter Registrierung (wie sie zum Beispiel im 18. Jahrhundert in Frankreich üblich war) und auf eine freie Handhabung sämtlicher Registrierungsmöglichkeiten. ${ }^{12}$ Adlung legt besonderen Wert auf Abwechslung ( $\$ 228$ und 237), kritisiert hingegen den ständigen Gebrauch immer gleicher Registerkombinationen (\$ 228).

Durch Agricolas Herausgebertätigkeit ergibt sich eine Verbindung zwischen Adlungs Publikation und dem Bach-Kreis. In seiner kritischen Stellungnahme zu Adlungs Ablehnung von Zungenregistern führt Agricola zweimal Bachs Vorliebe für diese an ${ }^{13}$ Adlungs übrige Ausführungen zur Registrierung bleiben unkommentiert. Daraus ist zu entnehmen, daß Agricola - obwohl sein eigener Essay einige signifikant abweichende Auffassungen enthält - sonst keine grundsätzlichen Vorbehalte gegen Adlungs Ansichten hatte. Bis vor kurzem bot diese Verbindung zwischen Agricola und Adlung den einzigen Zugang zu Bachs ebenso eigenwilliger wie meisterhafter Registrierkunst. ${ }^{14}$

Gruppe 2: Angaben über spezielle Registerkombinationen oder Anmerkungen zum Gebrauch bestimmter Register (ohne Bezug zu bestimmten Kompositionen).

In Verbindung mit dem Bau der Orgeln in Großhartmannsdorf und Fraureuth unterbreitete der Orgelbauer Gottfried Silbermann in Listenform Vorschläge zu Registerkombinationen, die auch Anweisungen sowohl für den Plenumklang („Volles reines Spiel“ oder „völligen Zug“) als auch für nicht-Plenumspiel enthielten. ${ }^{15} \mathrm{Da}$ die Kombinationen sich nicht auf bestimmte Werke beziehen, läßt sich nicht feststellen, für welche musikalische Form oder Satztechnik sie als passend galten. Überdies ist Silbermanns Beziehung zu Bach und dessen Kreis problematisch. Einerseits kannte Bach Silbermann und stand mit diesem verschiedentlich in geschäftlicher Verbindung. Auch gab er Konzerte auf den Silbermann-Orgeln der Dresdner Sophienkirche (1725 und 1731) und der Frauenkirche (1736); sein Sohn Wilhelm Friedemann Bach war von 1733 bis 1746 Organist an der Sophienkirche, und Agricola lobte bestimmte Eigenschaften von Silbermanns Instrumenten. ${ }^{16}$ Andererseits relativierte Agricola sein positives Urteil, indem er gewisse Beschränkungen kritisierte, ${ }^{17}$ und viele von Bachs Orgelwerken sind auf Silbermann-Orgeln wegen des begrenzten Pedalumfangs $\left(C, D-d^{\prime}\right)$ und wegen der Temperatur nicht spielbar. ${ }^{18}$ Auch war Sachsen, die Region, in der Silbermann seine Instrumente baute, für Bachs ursprünglichen Wirkungsbereich (Thüringen) von eher peripherer Bedeutung. Neuere

12 „Man soll also bald dies, bald jenes, gebrauchen; bald diese, bald jene zusammen ziehen. Dies aber kömmt aufs Gehör an, und nachdem die Einfälle sind, nachdem muß man ziehen. Man gehe demnach zuweilen alleine in die Kirche, und probire es so und so" (\$ 228).

13 Anm. zu $\S 104$ und $\S 267$.

${ }^{14}$ Dok III, Nr. 666 (Nekrolog), S. 88; J. N. Forkel, Ueber Johann Sebastian Bachs Leben, Kunst und Kunstwerke, Leipzig 1802 (Faks.-Ausgabe, Frankfurt/M. 1950), S. 20.

$15 \mathrm{Zu}$ diesen Listen sowie Dispositionen der betr. Orgeln vgl. E. Flade, Gottfried Silbermann, 2. Auflage, Leipzig 1953, S. 144 und 145.

16 Vgl. J. Adlung, Musica mechanica organoedi, Berlin 1768, Bd. 1, S. 212.

17 Ebd.

${ }^{18}$ Vgl. Dok II, Nr. 575. 
Untersuchungen bezweifeln denn auch, daß Silbermanns Instrument die ideale „Bach"-Orgel sei. ${ }^{19}$

Im Verlauf von Kapitel 8 seiner Musica mechanica organoedi gibt Adlung gelegentlich spezifische Registrierungsvorschläge:

$\S 216$ : „Z. Ex. ich habe bey der Quinte 6' das Principal $8^{\prime}$, nebst dem Bordun 16' und Rohrflöt 8', gehört, welches in der Höhe wohl lautete, aber in der Tiefe war die Quinte viel zu widrig anzuhören: wäre aber nur die Oktave $4^{\prime}$ noch dabey gewesen; so hätte man den Klang um ein merkliches verbessert."

$\S 218$ : Weit auseinanderliegende Register $\left(8^{\prime}+\right.$ Sesquialtera II $\left.; 16^{\prime}+2^{\prime} ; 16^{\prime}+1^{\prime}\right)$ ergeben einen schlechten Zusammenklang.

$\S 226$ : „Die Quintatön [16'] schlägt nicht gerne an, wenn man laufen will; also lasse man sie weg im vollen Werke, oder wenn man sonst geschwinde spielt. Besser ist ein Grob Gedackt, oder Bordun, wenn man dergleichen hat."

Eine Schrift des Berliner Garnisonkirchenorganisten Johann Friedrich Walther $^{20}$ beschreibt den Gebrauch der Register für die von Joachim Wagner $1724 / 25$ neuerbaute Orgel.

Drei Registrieranweisungen für die Orgel der Schloßkirche zu Lahm sind in einem Bericht über die Einweihung der Kirche und deren Orgel am 4. Mai 1732 erhalten. ${ }^{21}$ Deren Urheber ist nicht bekannt; möglicherweise stammen sie von Johann Lorenz Bach, einem Vetter Johann Sebastians und 1713-1717 dessen Schüler, der von 1718 bis zu seinem Tod im Jahre 1773 als Kantor und Organist in Lahm wirkte, oder von Heinrich Gottlieb Herbst, dem Erbauer der Orgel. Das Instrument ist in restauriertem Zustand erhalten und gilt als ein Musterbeispiel für den mitteldeutschen Orgeltyp. Die Registrieranweisungen waren für drei Choralvorspiele gedacht; ob es sich um ausgeführte Kompositionen oder um Improvisationen handelte, ist nicht bekannt, jedenfalls ist keinerlei zu dem Dokument gehörige Musik erhalten.

Von Bachs eigener Hand stammende Anmerkungen zur Registrierung enthält der Dispositionsvorschlag für den Umbau der Orgel in der Blasiuskirche zu Mühlhausen (1708): ${ }^{22}$ Anstelle der Oberwerk-Trompete soll ein 16'-Fagott angebracht werden, ,welcher ... in die Music sehr delicat klinget" (das heißt, bei der Ausführung des Continuo). ${ }^{23}$ An die Stelle des Oberwerk-Gemshorns soll

19 Siehe U. Dähnert, Johann Sebastian Bach's Ideal Organ, in: The Organ Yearbook 1, 1970, hier S. 23 f.; W. Schrammek, Versuch über Johann Sebastian Bachs Vorstellung von Orgelbau, Orgeldisposition und Orgelregistrierung, in: Johann Sebastian Bach und die Aufklärung, Leipzig 1982 (Bach-Studien. 7.), hier S. $201 \mathrm{f}$.

${ }^{20}$ Die, In der Königl. Garnison-Kirche zu Berlin befindliche Neue Orgel ..., Berlin 1727; vgl. H.-H. Steves, Der Orgelbauer Joachim Wagner (1690-1749), in: Archiv für Musikforschung 5, 1940, S. 23-26. J. S. Bach hatte wahrscheinlich während seines Besuchs am preußischen Hof im Jahre 1747 Gelegenheit, diese Orgel zu spielen; vgl. Dok II, S. 435.

${ }^{21}$ Siehe H. J. Busch, Unbekannte Registrierungsanweisungen aus dem Umkreis Johann Sebastian Bachs, in: Ars organi 33, 1985, S. 122-125.

${ }_{22}$ Dok I, S. $152 \mathrm{f}$.

${ }^{23}$ Johann Friedrich Walther schreibt, auf der Orgel der Garnisonkirche sei das Fagott 16' des [Haupt-]Manuals ,in der Music zu lauffenden Bässen nebst Zuziehung einer andern Stimme, schön zu gebrauchen“. Vgl. Steves, a. a. O., S. 24. 
eine „VioldiGamba $8 \mathrm{FuB}$ “ treten, „so da mit dem im Rückpositive vorhandenem Salicinal 4 Fuß admirabel concordiren wirdt.“"24

\section{Gruppe 3: Spezielle Registrieranweisungen für bestimmte Orgelkompositionen.}

Diese Quellengruppe liefert die präzisesten und detailliertesten Informationen. Leider sind sie - besonders im Blick auf Bach und seinen Kreis - nicht sehr zahlreich.

Von Bach beziehungsweise aus seinem Umkreis stammen folgende Quellen:

- Die Anweisung Organo pleno (oder ähnlich) zu Beginn einiger Bachscher Präludien und Fugen sowie einiger Choralbearbeitungen;

- die Anweisung à deux claviers et pédale in einigen Choralbearbeitungen;

- die Registrierung für "Gottes Sohn ist kommen“ (BWV 600) aus dem Orgelbüchlein: Manual Prinzipal 8', Pedal Trompete 8';

- Registrieranweisungen für Bachs Transkription von Vivaldis Concerto Grosso in d-Moll (BWV 596); hierbei handelt es sich allerdings um ungewöhnliche Registrierungen für ein ungewöhnliches Werk, die sich auf andere Orgelwerke Bachs kaum anwenden lassen;

- Anweisungen zu Registrierung und Manualgebrauch für die Choralbearbeitung „Ein feste Burg ist unser Gott“ (BWV 720); diese sind in der Handschrift Johann Gottfried Walthers überliefert und gehen möglicherweise nicht direkt auf Bach zurück;

- Bachs eigene Angaben zu einzelnen Stimmlagen (jedoch ohne konkrete Registrieranweisungen) für die Schübler-Choräle (BWV 645-650). ${ }^{25}$ In jedem Fall ist lediglich die Oktavlage angegeben (etwa in „Wachet auf, ruft uns die Stimme“, BWV 645: Destra 8 Fuß; Sinistra 8 Fuß; Pedal 16 Fuß);

- vereinzelte Anweisungen zu Manualwechseln in Bachs Werken ${ }^{26}$ sowie häufige Angaben zur Manualverteilung (Oberwerk, Rückpositiv) in Kompositionen Johann Gottfried Walthers. ${ }^{27}$

Detaillierte Registrieranweisungen für viele Stücke gibt der Merseburger Organist Georg Friedrich Kauffmann in seiner Sammlung Harmonische Seelenlust (Leipzig 1733-1736), die fast alle im frühen 18. Jahrhundert gebräuchlichen Typen von Choralbearbeitungen (Duette, Fughetten, verschiedene Arten von Cantus-firmus-Sätzen, darunter auch Trios) enthält. ${ }^{28} \mathrm{Obwohl}$ ein persönlicher Kontakt zwischen Kauffmann und Bach nicht nachweisbar ist, gibt es indirekte Hinweise darauf, daß Bach mit Kauffmann und dessen Schaffen vertraut war. ${ }^{29}$

${ }^{24}$ Walther (a. a. O.) bemerkt zum Salicinal $8^{\prime}$ im Seitenwerk der Garnisonkirchenorgel: „Diese Stimme ... klinget aber, wenn die ... Fugara $4 \mathrm{FuB}$ dazu gezogen, und damit lauffende Passagen gemacht oder Arpegiando gespielet ... wird, als wann mit dem Bogen, auf der Violine oder Viol di Gamba gestossen und gestrichen würde.“

${ }^{25}$ Diese stammen aus Bachs Handexemplar; vgl. C. Wolff, Bachs Handexemplar der Schübler-Choräle, BJ 1977, S. 120-129.

${ }^{26}$ Diskutiert bei G. Stauffer, Über Bachs Orgelregistrierpraxis, BJ 1981, S. 91-105.

${ }^{27}$ Siehe DDT 26/27, hrsg. von M. Seiffert.

${ }^{28}$ Vgl. Neuausgabe von P. Pidoux (Basel 1980), die aber Kauffmanns Anweisungen nicht immer getreu wiedergibt.

${ }^{29}$ Vgl. J. Rifkin, Artikel „Kauffmann, Georg Friedrich“, New GroveD, Bd. 9, S. 830 f. 
Daniel Magnus Gronau (ca. 1700-1747), Organist an der Danziger Johanniskirche, hinterließ für vier Folgen von Choralvariationen genaue Registrieranweisungen. ${ }^{30}$ Gronau gehört nicht zum Umkreis Bachs, und die von ihm genannten Register zeigen, daß er einen von der Thüringischen Orgel verhältnismäßig stark abweichenden Instrumententyp im Sinn hatte; wegen ihrer Genauigkeit sind die Anweisungen für Vergleichszwecke dennoch wertvoll.

\section{AGRICOLAS ABHANDLUNG VON 1757}

Die obige Aufschlüsselung deckt die Unzulänglichkeiten auf, die einen klaren Einblick in Bachs Registrierpraxis bisher verhinderten. Die ersten beiden Kategorien enthalten nur sehr wenig von Bach und seinem Kreis, es sei denn man ist bereit, aufgrund von Agricolas redaktioneller Tätigkeit auch Adlungs Ausführungen mitzurechnen. Adlungs Hinweise zu Registrierungen von nichtPlenumstücken sind jedoch vage und nicht schlüssig. Gruppe 3 ist wegen der Anwendung von spezifischen Registrieranweisungen auf bestimmte Stücke zwar potentiell am aufschlußreichsten, Belege aus dem direkten Bach-Umfeld liefert sie jedoch nur bruchstückhaft. Kauffmann und Gronau, deren präzise Registrieranweisungen für viele nicht-Plenumstücke ausführlich und informativ sind, gehören, wie bereits erwähnt, nicht zum unmittelbaren Bach-Kreis.

Agricolas Abhandlung ist eine wichtige Hilfe bei dem Versuch, das verschwommene Bild von Bachs Registrierpraxis schärfer zu fassen. Wie Adlungs Musica mechanica organoedi bezieht sich die Abhandlung auf die beiden ersteren Quellengruppen, indem sie sowohl generelle Prinzipien und Bemerkungen zur Registrierung als auch spezifische Registerkombinationen und Hinweise zum Gebrauch von bestimmten Registerzügen mitteilt. Tatsächlich jedoch bildet der Essay eine Kategorie für sich, da er die einzige substantielle Abhandlung über Registrierung ist, die mit Sicherheit aus dem Kreis um Johann Sebastian Bach stammt. So fungiert er einerseits als Objektiv, das eine schärfere Sicht auf die bruchstückhaften Belege aus Bachs Umfeld gestattet, andererseits dient er als Prisma, durch das die spezifischeren Zeugnisse von außerhalb des Kreises gefiltert und auf ihre mögliche Relevanz für die Konzepte von Bach und seinen Schülern geprüft werden können.

Der Essay besteht aus vier Teilen:

I. Kurze Würdigung der Sammlung einiger Nachrichten (S. 486-487)

II. Beschreibung von in zeitgenössischen Orgeln zu findenden Registern (S. 487-501)

III. Informationen über Registerkombinationen (S. 501-505)

IV. Dispositionen von sechs Orgeln (S. 506-518): Petrikirche zu Freiberg, Stiftskirche zu Halberstadt, Marktkirche zu Halle, Domkirche zu Königsberg, Johanniskirche zu Magdeburg und Stadtkirche zu Meerane.

${ }^{30}$ Vier Choralvariationen für Orgel, hrsg. von G. Frotscher, Augsburg und Kassel 1927. Diese Stücke bilden lediglich einen Bruchteil von Gronaus Schaffen, das im Zweiten Weltkrieg fast völlig verloren ging. 


\section{Der Text von Teil III lautet folgendermaßen:}

„Nachdem wir nun die Stimmen der Orgeln so viel möglich beschrieben haben: so möchte es einigen Lesern nicht unangenehm seyn, wenn sie auch von der Art, wie man diese Stimmen brauchen und mit einander verbinden soll, etwas zu lesen bekämen. Da die Veränderungen hierbey so unzählig sind, daß es nicht möglich ist, sie alle anzuführen; da auch über dieses einem Orgelspieler, der ein feines Gehör hat, nicht schwer werden wird, ihre Wirkungen selbst auszuprobiren; so werden etliche allgemeine Anmerkungen hinlänglich seyn, einem, der im Ziehen der Orgelregister noch nicht recht erfahren ist, zu weitern Versuchen Anlaß zu geben.

Wenn man recht stark spielen will; so zieht man das volle Werk, zu welchem alle oben beschriebenen Principalstimmen gehören. Diesen kann man noch die Trompeten von 16.8 und $4 \mathrm{FuB}$, wenn sie rein gestimmet sind, beyfügen; man koppelt auch wohl ein anders Clavier, auf welchem gleichfals das volle Werk gezogen ist, dazu. Hierauf kann man nicht nur langsam, sondern auch, wenn die Orgel gut anspricht, und die Finger es erlauben wollen, geschwinde Sachen spielen. Doch muß die Vollstimmigkeit hierbey vorzüglich herrschen. Die französischen Organisten ziehen die Rohrwerke nicht mit zum vollen Werke, weil sie, wenn man in der Tiefe mit vollen Griffen spielt, gar zu widrig klingen. Man muß sich ja aber überhaupt aller solcher Griffe, auf der Orgel, wenn 16 oder 8füßige Stimmen gezogen sind, enthalten. Das Flötenwerk wird bey dem vollen Werke nicht mit gezogen. Außer wenn das Principal nur achtfüßig ist; so kann und muß ein 16 füßig Gedackt, Bordun Quintadena oder Rohrflöte dazu gezogen werden. Ein 16füßiger Bordun erhebt die Gravität auch eines 16füßigen Principals sehr. Ein gleiches ist zu beobachten, wenn das Principal nur 4füßig ist: da denn nothwendig ein 8füßiges Flötenwerk, als die Fundamentalstimme, dazu gezogen werden muß.

Eine 4füßige Stimme kann nicht ohne die Bedeckung einer achtfüßigen gebrauchet werden; man müßte denn sehr geschwinde Passagien darauf spielen.

Die Mixturwerke können gar nicht ohne Begleitung der andern Principalstimmen gebrauchet werden. Sind aber diese dabey; so füllen jene vortreflich aus, und die Uebelklänge, welche so viel gegen einander schlagende Accorde verursachen würden, werden, weil die Mixturen aus lauter kleinen Pfeifen bestehen, von jenen bedecket, und gleichsam verschlungen. Zum Flötenwerke gehören die Mixturen gar nicht. Doch kann ein 8 füßig Gedackt zur Sesquialtera mit der 1 füßigen Octave, bey geschwinden Brechungen, gezogen werden.

Eine Quinte oder Terze muß allezeit eine noch höhere Octave oder Superoctave über sich haben. Z. E. ist die Quinte von $3 \mathrm{Fuß}$, so muß nebst dem 8 und 4 füßigen Register auch noch eine 2 füßige Stimme gezogen werden.

Man läßt nicht gern eine Octave in der Mitten aus. Z. E. ein 8 und 2 füßiges Register, ohne

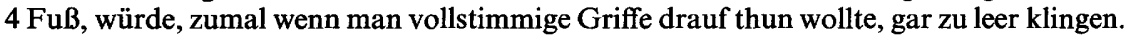
Spielt man aber nur einstimmig auf einem Claviere, z. E. in einem Trio; so kann man gar wohl 16 und $4 \mathrm{Fuß}$ mit einander vereinigen. So thut z. E. Quintadene $16 \mathrm{Fuß}$, und Hohl= oder Waldflöte $4 \mathrm{Fuß}$, in diesem Falle, gute Wirkung. Auch so gar der 16 füßige Bordun mit dem 1 füßigen Sifflet, thut gute Wirkung, wenn man einstimmige geschwinde Passagien darauf spielet. Ueberhaupt kömmt es bey Ziehung der Register viel darauf an, ob man einstimmig oder vielstimmig auf einem Claviere spielen will.

Ein Rohrwerk wird selten allein gebraucht. Man nimmt immer eine gleichtönige Stimme vom Pfeifenwerk dazu, welche das Schnarren des Rohrwerks bedecket. So gehöret z. E. zur 8 füßigen Trompete das 8 füßige Principal. Die Vox Humana muß, wo ja irgend noch einige Gleichheit mit der Menschenstimme herauskommen soll, immer, wo nicht das Principal (wie Herr Silbermann verlangte,) doch wenigstens das 8 füßige Gedackt oder Rohrflöte bey sich haben. Am allerbesten aber schickt sich dazu die 8 füßige Hohlflöte, wenn man sie haben kann. Doch kann man ein 8 füßig Rohrwerk auch mit einem 4 füßigen 
Pfeifenwerk, und umgekehrt, wohl gebrauchen. Auch kann ein dergleichen Rohrwerk mehrern höhern Stimmen bequem zum Fundamente dienen.

Soll eine Stimme des auszuführenden Stücks hervor ragen; so müssen auf dem Claviere, auf welchem man sie ausführet, stärkere Register angezogen werden, als auf dem andern.

Das Pedal muß sich nach der Stärke des Manuals richten.

Die Franzosen spielen ihre Fugen auf dem 8 füßigen Rohrwerk, mit einem tiefern Bordun und einer höhern Octave. Sie glauben, daß man, wenn keine Mixturen dabey sind, die Eintritte der Stimmen deutlicher wahrnehmen könne; und vielleicht haben sie hierinn nicht unrecht. Die beyden zum Rohrwerke gezogenen Principalstimmen nennen sie le fond de la Trompette, ou du Cromhorne.

Die sogenannte Tierce en taille bey den Franzosen, dergleichen man in ihren Orgelbüchern viele findet, wird aus folgenden Stimmen zusammen gezogen: Bordun 8 FuB, Octave 4 $\mathrm{FuB}$, Nasat $3 \mathrm{FuB}$, Tertie $1 \frac{1}{2} \mathrm{FuB}$, und Octave $2 \mathrm{FuB}$; wozu man auch noch ein 16 füßig Gedackt nehmen kann. Auf dem andern Claviere, auf welchen man die Begleitungsstimmen ausführet, zieht man ein 8 füßiges Principal allein, oder ein paar Flöten, und im Pedal Principal Subbaß 16, und Octave 8 Fuß.

Die Alten glaubten, daß zwo gleiche Stimmen von verschiedener Mensur übel klingen müßten, wenn sie zusammen gezogen würden. Sind diese Stimmen gut gearbeitet und rein gestimmet; so kann man die Alten alle Tage durch derselben vereinigten Gebrauch widerlegen. Ich habe in einer gewissen Orgel das liebliche Gedackt, die Vugara, die Quintadene und die Hohlflöte alle von $8 \mathrm{Fuß}$, ohne irgend eine andere Stimme, zusammen gehöret, welches eine schöne und fremde Wirkung that."

\section{ANALYSE UND VERGLEICH}

\section{Das Plenum}

Sowohl Mattheson als auch Adlung verlangen, daß für das Manual-Plenum neben den Prinzipalen und Mixturen alle Labialregister - einschließlich Flöten, Streicher- und Aliquotstimmen - gezogen werden. Silbermann fordert in seinen Plenum-Vorschlägen Flöten und Prinzipale, scheut aber die Flöten-Aliquotstimmen (beispielsweise Cornett). Agricola dagegen hält sich an die Anleitungen früherer Autoren, wie Praetorius ${ }^{31}$ und Werckmeister ${ }^{32}$, nach denen das Plenum nur aus Prinzipalstimmen, unter Ausschluß von Flöten und Streicherstimmen gebildet werden soll. ${ }^{33}$ Merkwürdigerweise ließ Agricola Adlungs Anweisungen zur Zusammensetzung des Plenum unkommentiert. Vielleicht war er sich dessen bewußt, daß seine Vorstellungen in diesem Punkt konservativ waren und nicht zu seiner zukunftsweisenden Billigung mehrerer gleichzeitig gezogener $8^{\prime}$ Stimmen paßten.

${ }^{31}$ M. Praetorius, Syntagma musicum, Bd. 2, Wolfenbüttel 1619 (Faks.-Ausgabe, Kassel 1958), S. 101 und 113.

${ }^{32}$ A. Werckmeister, Erweiterte und verbesserte Orgel-Probe, Quedlinburg 1698, S. 72; Musicae mathematicae hodegus curiosus, Frankfurt und Leipzig 1686, S. 52f. Werckmeister rät in diesen Schriften von dem Gebrauch mehrerer Stimmen in derselben Lage ab.

${ }^{33}$ An einer früheren Stelle seines Essays erläutert Agricola, daß er alle Labialstimmen mit Ausnahme der Prinzipale zu den Flöten zählt; wenn er also schreibt, „das Flötenwerk wird bey dem vollen Werke nicht mit gezogen“ (S. 502), meint er sowohl Flöten- als auch Streicherstimmen. 
Die Eingangssätze von Gronaus vier Choralvariationen (allesamt ausgedehnte einmanualige, kontrapunktische Gebilde) scheinen für das Plenum bestimmt zu sein, enthalten jedoch keine Registrieranweisungen. Gronau gibt keine PlenumVorschriften, doch mehrere Variationen verlangen das Solopedal-Plenum, das wie bei Mattheson und Agricola alle Labialregister enthält.

\section{Gronau}

Ein feste Burg: Princip. 16', Basso coperto 16', Violone 16', Octava 8', Flauto 8', Octava 4', Quinta 3', Octava 2', Mixtura, Trombone 16', Tromba 8', Cornetto 4'

Was Gott thut das ist wohlgetan: Princip. 16', Violone 16', Basso coperto 16', Octava 8', Flauto 8', Octava 4', Mixtura

In seiner Anweisung, alle Labiale zu ziehen, stimmt Gronau ganz mit Mattheson und Adlung überein, in der Verwendung des Rohrwerks im Pedalplenum scheint er jedoch flexibler zu sein als diese. Obwohl Kauffmann mehrfach das Plenum („das volle Werk“) fordert, gibt er nirgends Anleitungen für dessen Zusammensetzung. Von besonderem Interesse sind jedoch die Registrierungen, die er alternativ zum Plenum vorschlägt.

\section{Kauffmann}

Ein feste Burg: Principal $8^{\prime}+4^{\prime}$, oder Gedact $8^{\prime}+$ Princip. $4^{\prime}$; item das volle Werck Es ist das Heil: Gedact, Spitzflöth, Viola; item das volle Werck

In dich hab ich gehoffet, Herr: Trompeta + Princip. $8^{\prime}+$ Octav 4'; oder das volle Werck Nun lob, mein Seel, den Herren: Principal $8^{\prime}+$ Octava $4^{\prime}+$ Super Octav $2^{\prime}$; item das volle Werck

Darüber hinaus gibt es bei beiden Komponisten Stücke, die von ihrer Satztechnik her für das Plenumspiel geeignet wären, die aber ausdrücklich anders bezeichnet sind:

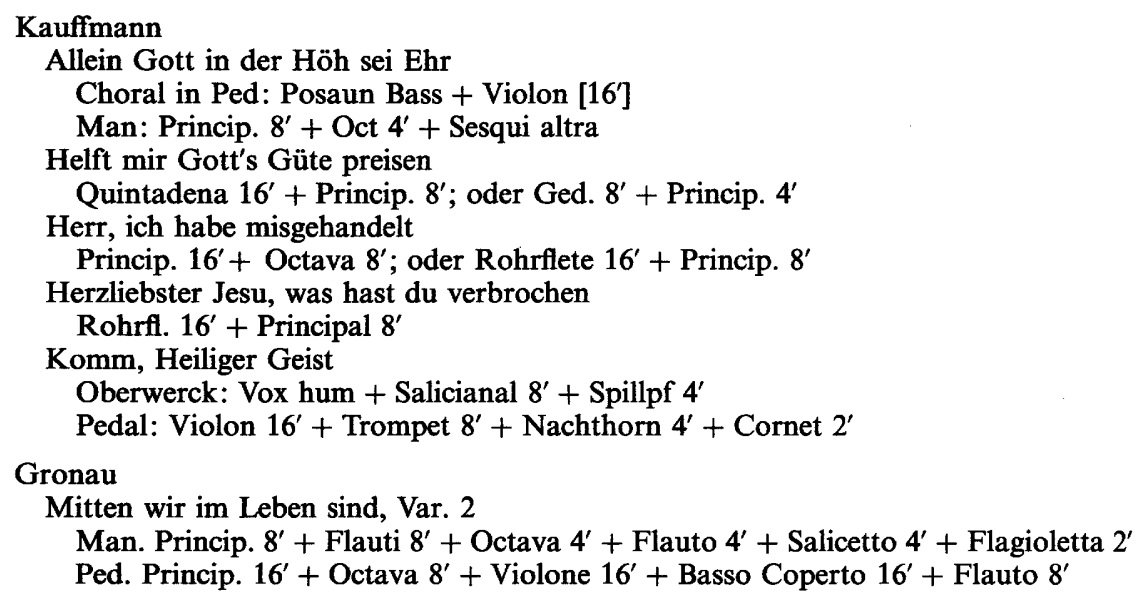

Bei beiden Organisten gibt es anscheinend eine beachtliche Bandbreite in der Anwendung des Plenum, zumindest in choralgebundenen Werken; das Plenum wird durch keine spezifische Form oder Satztechnik zwingend gefordert und oft sind alternative Registrierungen möglich. 


\title{
2. Zusatz von Trompetenstimmen zum Plenum
}

Im Gegensatz zu Mattheson und Adlung erlaubt Agricola den Ausbau des Plenums durch manualiter-Trompetenstimmen. Seine Abhandlung scheint für diese Praxis den frühesten unmißverständlichen Beleg abzugeben. ${ }^{34}$ Die Gründe für das langanhaltende Schweigen ${ }^{35}$ bezüglich der Verwendung von manualiterZungenstimmen im Plenum sind alles andere als klar; eine mögliche Ursache deutet Agricola mit der Bemerkung an, „wenn sie rein gestimmet sind“"(S. 502). Adlung zum Beispiel klagt in seiner Musica mechanica organoedi $i^{36}$ über die ständig verstimmten Rohrwerke und empfiehlt, in neuen Orgeln ihre Zahl drastisch zu vermindern (mit Ausnahme der obligatorischen Bestückung des Pedals). Agricola dagegen beteuert in einer redaktionellen Fußnote, Bach sei „ein großer Freund davon“ gewesen, und rügt diejenigen Organisten, die zu bequem sind, sie in guter Stimmung zu halten. ${ }^{37}$

Im Unterschied zu Kauffmann erwähnt Gronau Trompetenstimmen kein einziges Mal. Kauffmann verwendet Kombinationen mit Einbeziehung von Rohrwerken recht großzügig, und dies sogar in Stücken, deren Satztechnik den Gebrauch des Plenum nahelegen würde.

\author{
Kauffmann \\ In dich hab ich gehoffet Herr \\ Trompeta + Princip. $8^{\prime}+$ Octav $4^{\prime}$; oder das volle Werck \\ Komm, Heiliger Geist \\ Oberwerck: Vox hum + Salicianal $8^{\prime}+$ Spillpf $4^{\prime}$ \\ Pedal: Violon $16^{\prime}+$ Trompet $8^{\prime}+$ Nachthorn $4^{\prime}+$ Cornet $2^{\prime}$
}

\section{Der Sechzehnfuß im Manualplenum}

Wie Adlung (und vermutlich auch Mattheson) befürwortet Agricola eine solide 16'-Basis für das Manualplenum, wobei er sogar für eine Ergänzung des Prinzipal 16' durch einen Bordun 16' plädiert. Alle Quellen teilen eine Vorliebe für die 16'-Lage im Plenum oder in vom Plenum abgeleiteten Kombina-

${ }^{34}$ Man beachte jedoch, daß Christian Ludwig Boxberg in seiner Ausführlichen Beschreibung Der Grossen Neuen Orgel In der Kirchen zu St. Petri und Pauli allhie zu Görlitz, Görlitz 1704, S. 13, über die 1703 errichtete Casparini-Orgel bemerkt: „So kan man auch, um das Ripieno noch stärcker zu machen, alle Fleuten, weit-mensurirte Register und Schnarr-Wercke mit gebrauchen, ohne, daß sie ihrer ungleichen mensur wegen mit denen Praestant-Registern eine disharmonie verursachen solten." Weiter unten auf derselben Seite schreibt er: „Bombart 16. Fuß ist ein sehr starckes Schnarr-Werck, so ... das volle Werck gar um ein sehr merckliches verstärcket.“ Da Boxberg die Görlitzer Orgel jedoch für ein ungewöhnliches und einmaliges Instrument erklärt, erscheint es unklug, seine Ausführungen so kategorisch zu verstehen wie diejenigen Agricolas.

${ }^{35}$ Walther zum Beispiel rühmt die „Trompett 8 Fuss“ im Seitenwerk der Garnisonkirchenorgel und gibt eine detaillierte Beschreibung von deren Beschaffenheit, bemerkt dann jedoch knapp, sie werde für „Veränderungen“ gebraucht und klinge gut zusammen mit dem Principal 8', besonders "wenn mit denen Paucken pedaliter accompagniret wird“" (Steves, S. 25).

${ }^{36}$ Bd. $1, \S 104$ und $\S 267$.

${ }^{37}$ Anmerkung zu Bd. 1, §104; vgl. auch die Anmerkung zu § 267. 
tionen. ${ }^{38}$ Kauffmann schätzte offensichtlich den $16^{\prime}$-Klang, oft in Kombinationen mit Registern lediglich in 8 '-Lage:

Kauffmann

Herr, ich habe misgehandelt

Princip. 16' + Octava $8^{\prime}$ oder Rohrflete $16^{\prime}+$ Princip. $8^{\prime}$

Helft mir Gott's Güte preisen

Quinta dena $16^{\prime}+$ Princip. 8' oder Ged 8' + Princ. $4^{\prime}$

Treuer Gott, ich muß dir klagen

Princip. 16' + Gedact $8^{\prime}+$ Spill flöth $4^{\prime}$

Schönster Immanuel

Quinta dena 16' + Principal 8' oder Princip. 8' allein

Tatsächlich verwenden 25 der 51 von Kauffmann mitgeteilten Registrierungen (also annähernd die Hälfte) den 16'-Klang auf einem oder mehreren Manualen. Adlung ( $\$ 216)$ betont:

ich habe bey der Quinte 6' das Principal 8', nebst dem Bordun 16' und Rohrflöt 8', gehört, welches in der Höhe wohl lautete, aber in der Tiefe war die Quinte viel zu widrig anzuhören: wäre aber nur die Oktave 4' noch dabey gewesen; so hätte man den Klang um ein merkliches verbessert.“

Eine der Orgelchoral-Registrierungen in Lahm verlangt die folgenden Züge im Hauptwerk:

Quintatön 16' + Principal 8' + Gedact 8' + Octav 4'.

Die thüringischen Orgeln des 18. Jahrhunderts verfügten in der Tat über eine Reihe von 16'-Registern, wie sie in solcher klanglichen Klarheit und Schärfe in modernen Orgeln normalerweise nicht zu finden sind ${ }^{39}$ Doch Unterschiede in Mensur und Stimmqualität erklären die ausgeprägte Vorliebe des 18. Jahrhunderts für den 16'-Klang nur zum Teil; in erster Linie dürfte dafür ein anderes Klangideal verantwortlich sein. Adlung (\$ 231) stimmt in der Empfehlung, stets mehr als einen 16' zu verwenden, mit Agricola überein, und auch Gronau folgt dieser Richtlinie.

\section{Gronau}

Ein feste Burg, Var. 1 (Baß auf separatem Manual)

Principal 16' + Octava $8^{\prime}+$ Flauto + Fagotto $16^{\prime}$

Mitten wir im Leben sind, Var. 3

Manual: Principal 16' Quintadena $16^{\prime}+$ Octava $8^{\prime}+$ Quintadena $8^{\prime}+$ Flauti $8^{\prime}+$ Flauto $4^{\prime}+$ Octava $4^{\prime}+$ Octava $2^{\prime}$

${ }^{38}$ Silbermanns Anleitungen für das Plenumspiel konnten keine $16^{\prime}$-Stimmen enthalten, da die Orgeln, für die sie intendiert waren, keine besaßen.

39 An einer früheren Stelle seines Essays beschreibt Agricola zum Beispiel ein von ihm besonders geschätztes 16'-Register: „In der Schloßorgel zu Altenburg ist eine Querflöte von $16 \mathrm{Fuß}$.... Sie ist ein enges offenes Pfeifenwerk von eben der Mensur, als die in dieser Orgel befindliche 8 füßige überaus schöne Viola da Gamba. Und wenn sie mit dieser zusammen gezogen, und darauf ... mit geschwinden Läufen und Brechungen gespielet wird, so thut dieses eine sehr schöne Wirkung; und die in diesen beyden Stimmen befindliche angenehme Schärfe, kömmt dem Schneiden des Bogenstrichs auf einem Baßinstrumente so nahe, als es nur durch Pfeifen möglich zu machen ist" (S. 497). 
Es wird schier der letzte Tag herkommen, Var. 1

Manual: Principal 16' Quintadena 16' + Octava $8^{\prime}+$ Flauti $8^{\prime}+$ Octava $4^{\prime}+$ Octava $2^{\prime}$

\section{Terzen in Mixturen und im Plenum}

Agricola $^{40}$ und auch Adlung ( $\$ 233$ und $\S 244$ ) setzen das Vorkommen von Terzen in Mixturen als selbstverständlich voraus. Ferner verlangen sowohl Adlung als auch Mattheson die Sesquialtera im Plenum. Kauffmann macht zwar zur Zusammenstellung des Plenum keine Angaben, doch auch er war der Verwendung von Terzen in Plenum-bezogenen Registrierungen nicht abgeneigt.

Kauffmann

Allein Gott in der Höh' sei Ehr

Choral in Ped: Posaun Bass [16'] + Violon [16']

Man: Princip $8^{\prime}+$ Octav $4^{\prime}+$ Sesqui altra

Die Wagner-Orgel der Berliner Garnisonkirche enthielt - laut Johann Friedrich Walther - ebenfalls Terzen, und zwar im Scharff (der tiefsten Mixtur) des Manuals wie auch des Seitenwerks. Walther vermerkt überdies, das fünffache Cornett $\left(\mathrm{c}^{\prime}-\mathrm{c}^{\prime \prime}\right)$ im Manual habe „eine etwas weite mensur, und giebt dem Vollenwercke, eine schöne force" (Steves, S. 23). Mit seiner Ablehnung von Terzen sowohl in Mixturen als auch im Plenum steht Silbermann allein (dies ist wahrscheinlich eine Folge seiner Ausbildung in Frankreich, wo weitmensurierte Cornett- und engmensurierte Prinzipalstimmen gewöhnlich nicht gemischt wurden). Begreiflicherweise machten in vielen von Johann Sebastian Bach gespielten und geschätzten Orgeln die Terzen in den Mixturen deren Ausschluß vom Plenum unmöglich.

Einige der geschilderten Praktiken sind auch heute noch strittig, beispielsweise hinsichtlich eines gleichbleibend starken Anteils von 16'-Registern im Manualplenum oder der häufigen Einbeziehung von (prinzipal-mensurierten) Terzregistern in das Plenum. Als gesichert kann jedoch gelten, da $\beta$ keine von diesen Verfahrensweisen Bachs Plenumkonzeption völlig fremd war. Was wir darüber allerdings nicht wissen, ist das Ausmaß, in dem das Plenum gewöhnlich verwendet wurde. Soll es grundsätzlich für alle Präludien und Fugen benutzt werden ${ }^{41}$ also etwa auch für Präludium und Fuge A-Dur BWV 536, für die Fugen D-Dur BWV 532 und G-Dur BWV 577? Wenn nicht, welches sind die Alternativen? Kann ein zweites Manualplenum als gleichwertig fungieren oder ist das normale Plenum immer das des Hauptwerks? Sollen die freien Orgelwerke - falls nicht anderweitig spezifiziert - durchweg mit derselben Plenum-

\footnotetext{
40 S. 492.
}

${ }^{41}$ Die vorhandenen Quellen stimmen darin überein, daß freie Werke mit dem Plenum gespielt werden sollen; die Quellen sind allerdings weder sehr zahlreich noch völlig eindeutig. Vgl. Stauffer, S. $92 \mathrm{f}$. 
registrierung gespielt werden $?^{42} \mathrm{Muß}$ man die ausgedehnten Choralbearbeitungen mit dichter Satztechnik (etwa die dreiteilige Bearbeitung von „O Lamm Gottes unschuldig“" aus den Leipziger Chorälen, BWV 656) ausschließlich auf dem Plenum spielen, oder bieten weniger aggressive Registrierungen eine legitime Alternative? Kurz, wir wissen nicht, wie erfindungsreich und flexibel Bach und sein Kreis in der Registrierung von Werken waren, die moderne Organisten normalerweise durchweg im Plenum spielen.

\section{Der Vierfuß als tiefste Lage}

Den Klang eines 4'-Registers ohne mitgehenden Achtfuß lehnt Agricola ab, es sei denn, „man müßte ... sehr geschwinde Passagien darauf spielen“ (S. 502). Welche Art von Satzbild Agricola hier genau vor Augen hat, bleibt undeutlich. Meint er galante Werke in der Art von Haydns Flötenuhrstücken oder denkt er an ältere Kompositionen von eher komplexer Faktur wie den zweiten Satz von Bachs Pastorale (BWV 590)? Weder Kauffmann noch Gronau liefern Beispiele für ein 4'-Register ohne mitgehenden Achtfuß. Zwar verlangen einige von Kauffmanns Kombinationen (fast immer bei Triosätzen) nur ein einzelnes 4'-Register, doch bedeutet dies entsprechend seinem Vorwort stets, daß die betreffende Stimme eine Oktave tiefer zu spielen ist. Ein ähnlicher Fall findet sich in Bachs Registrieranweisungen für die Schübler-Choräle. Die PedalCantus-firmi in BWV 646, 647 und 650 enthalten die Anweisung „4 Fuß“, doch dient dies lediglich dazu, den Choral in die Tenorlage zu plazieren. ${ }^{43}$

\section{6. „Offene“ beziehungsweise „Lücken“-Registrierungen}

Der Gebrauch von „Lücken“-Registrierungen $\left(16^{\prime}+4^{\prime}, 8^{\prime}+22 / 3^{\prime}\right.$ und ähnlich) stellt weiterhin eines der größten Probleme der deutschen Registrierungspraxis des 18. Jahrhunderts dar; und auch Agricola trägt wenig zu seiner Klärung bei. Im allgemeinen bewertet Agricola derartige Registrierungen eher negativ (besonders für akkordisches Spiel), doch lehnt er sie nicht völlig ab. Für einzelne Melodiestimmen, etwa in einem Trio, scheint er die Kombination $16^{\prime}+4^{\prime}$ gegenüber $8^{\prime}+2^{\prime}$ zu bevorzugen; dies bezieht sich insbesondere auf den Diskant im Manual. Verglichen mit anderen Quellen scheint Agricolas Ratschlag ein wenig extrem. Adlung beispielsweise ist im Gebrauch von „Lücken“Registrierungen zurückhaltend ( $(218)$, besonders in Verbindung mit Aliquoten, doch bei Oktavlücken ist er weniger reserviert (und würde wohl auch $8^{\prime}+2^{\prime}$ tolerieren).

„Wollen doch nicht einmal die Octavenstimmen wohl lauten, wenn sie allzuweit von einander entfernet sind. Z. Ex. $16^{\prime}$ zu $2^{\prime}$ oder $1^{\prime}$.“

${ }^{42}$ Stauffer gibt ein überzeugendes Plädoyer für diese Praxis, seine Argumentation beruht jedoch auf einem Indizienbeweis, denn bislang sind keine eindeutigen und detaillierten Instruktionen bekannt geworden.

43 In Bachs Handexemplar findet sich für BWV 650 die zusätzliche Anweisung „Ped. 4 f.u. eine 8tav tiefer" (in modernen Ausgaben nicht enthalten); dies plaziert den Cantus firmus in die Tenorlage. Vgl. Wolff, a. a. O., S. 126 und 129. 
Von den in Gruppe 3 genannten Quellen scheint Kauffmann mit seiner Vorliebe für den 16'-Klang und seiner Abneigung gegen „Lücken“-Registrierungen Agricolas Einstellung am nächsten zu stehen. In nicht weniger als vier Fällen gibt Kauffmann die Registrierung Fagott 16', Quintadena $8^{\prime}$, Spitzflöte $2^{\prime}$ an eine Kombination, die vielleicht eher einer Eigenart seines Geschmacks entsprang oder auf den ihm bekannten Instrumenten einen besonderen Wohlklang hervorbrachte, als daß sie eine generelle Wertschätzung dieser Klangverbindung im frühen 18 . Jahrhundert kennzeichnet. Zwei weitere Kombinationen können kaum als extreme Beispiele von „Lücken“-Registrierungen gewertet werden und scheinen nicht auf eine besondere Bevorzugung der eigentümlichen Klangfarbe hinzudeuten.

\section{Kauffmann}

Nun danket alle Gott (alio modo)

Hauptmanual: Gedackt $8^{\prime}+$ Nassat + Spitz Flöth $2^{\prime}$

Nun ruhen alle Wälder

Oberwerck: Voxh + Rohrfl. $8^{\prime}+$ Rohrflöte $2^{\prime}$

Gronau führt lediglich drei „Lücken“-Registrierungen an:

\section{Gronau}

Ein feste Burg, Var. 1

2. Clav.: Principal 4' + Flauto $4^{\prime}+$ Octava $1^{\prime}+$ Regale $8^{\prime}$

Was Gott thut, das ist wohlgethan, Var. 1

Solo (Tenor): Octava $8^{\prime}+$ Quintadena $8^{\prime}+$ Flauto $8^{\prime}+$ Octava $2^{\prime}$

Was Gott thut, Var. 3

Quintadena $16^{\prime}+$ Flauto $4^{\prime}$

Die letztgenannte Registrierung erinnert an Agricola; hier wird sie allerdings nicht auf eine einzelne Triostimme, sondern auf einen dreistimmigen Manualitersatz angewendet.

Andere Quellen zeigen eine deutlich größere Vorliebe für „offene“ Registrierungen als Agricola. Silbermanns Vorschläge $\left(8^{\prime}+4^{\prime}+1^{\prime}\right.$ oder $8^{\prime}+2^{\prime}$ oder $\left.8^{\prime}+22 / 3^{\prime}+13 / 5^{\prime}\right)$ sind vielleicht am ehesten bekannt. Tatsächlich könnte die heutige Wertschätzung solcher Registrierungen sehr wohl auf Silbermanns Empfehlungen zurückgehen, ${ }^{44}$ die ja seit vielen Jahren veröffentlicht sind und oft zitiert werden. Doch auch weniger bekannte Quellen billigen derartige Registrierungen, zum Beispiel Johann Friedrich Walther in Berlin (Steves, a. a. O., S. 24).

Manual: „Octave 2 Fuß ... kan zu einer 8füßigen Stimme allein ... gebrauchet werden.“ Seitenwerk: "Waldfloet 2 Fuß lässet sich mit dem Principal [8'] oder auch gedact 8 Fuß gar artig hören."

Seitenwerk: „Siffloit 1 Fuß, ist ... mit einer achtfüßigen [Stimme] zur Veränderung, wie auch im vollen Wercke mitzuziehen und zu gebrauchen."

${ }^{44}$ Dies mag teilweise auch dadurch bedingt sein, daß Silbermanns Registrierungen sehr gut mit der neoklassischen Vorliebe für helle, klare Klangfarben korrespondieren, die zu Beginn dieses Jahrhunderts bestand. 
Sowohl bei Kauffmann als auch bei Gronau finden sich Triosätze, mit instruktiven Registrierungen. Kauffmanns Vorschläge sind häufig traditionell und konservativ.

\author{
Kauffmann \\ Jesus Christus unser Heiland \\ Manual: Principal $8^{\prime}$ \\ Rückpositiv: Principal 4' [eine Oktave tiefer gespielt] \\ Pedal: Sub Bass $16^{\prime}+$ Octaven B. $8^{\prime}$
}

Ähnliche Registrierungen werden in drei weiteren Fällen verlangt: „Wo Gott zum Haus nicht gibt sein Gunst“, „Gelobet seist du, Jesu Christ“" und „Herr Gott, dich loben alle wir". Doch Kauffmann schlägt auch einige weniger orthodoxe Möglichkeiten vor:

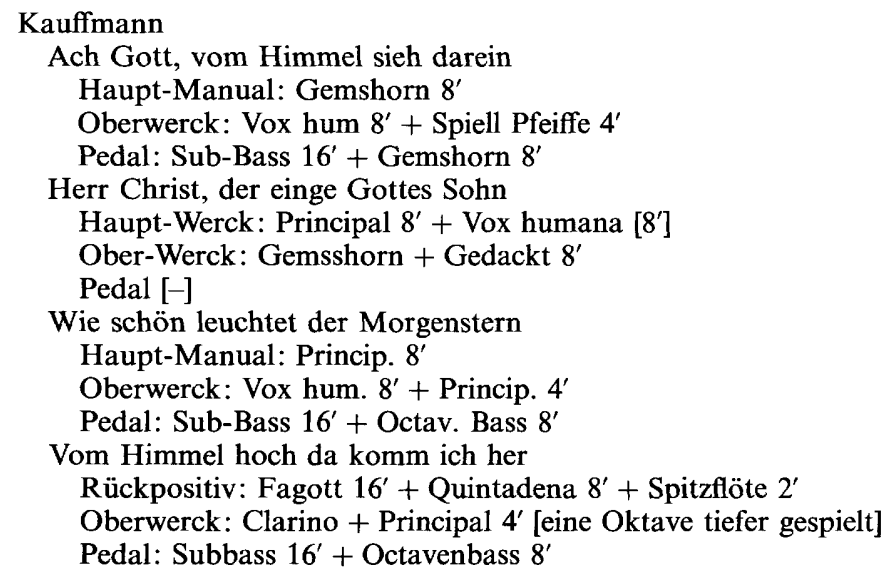

Im letzten Beispiel beruht eine Diskantstimme (vermutlich die obere) auf einem 16'-Klang, vergleichbar mit Agricolas $16^{\prime}+4^{\prime}$; eigentümlicherweise ist dies zugleich Kauffmanns einziger Triosatz mit „Lücken“-Registrierung. Nicht für jeden Triosatz gibt Kauffmann eine Pedalregistrierung, doch wo er dies tut, ist sie ausnahmslos auf einem Subbaß $16^{\prime}$ gegründet.

Bach dürfte gegen eine $16^{\prime}+4^{\prime}$ Registrierung des Manuals nichts einzuwenden gehabt haben, vermutlich hätte er sie sogar gutgeheißen. Seine Registrieranweisungen in den Schübler-Chorälen jedoch stellen Agricolas Registrierung für Trios in Frage.

Bach

Wachet auf, ruft uns die Stimme (BWV 645)

Destra $8^{\prime}$, Sinistra $8^{\prime}$, Pedal $16^{\prime}$

Wo soll ich fliehen hin (BWV 646)

1. Clav. 8', 2. Clav. 16' [Baßstimme], Ped. $4^{\prime}$

Bei beiden Stücken handelt es sich um Trios, das erste mit einem ChoralCantus-firmus im Tenor (linke Hand), das zweite mit diesem in der Tenorlage (Pedal). Keine der beiden Registrieranweisungen schließt den Gebrauch einer 
„Lücken“-Registrierung von vornherein aus (vorausgesetzt, die angegebene Lage läßt die Verbindung mit einer höheren Lage überhaupt $\mathrm{zu}$ ), doch gibt es keinen Hinweis auf einen Sechzehnfuß als tiefste Lage für eine Oberstimme. Vielmehr erklingen die Oberstimmen, ganz gleich ob sie im Manual oder im Pedal liegen, stets in $8^{\prime}$-Lage.

\section{Verbindung von Labialregister und Zungenstimme}

Nach Agricola wird ein Rohrwerk in Achtfußlage selten allein, sondern meist zu einer 8'-oder 4'-Labialstimme gezogen. Dem scheint auf den ersten Blick Bachs Anweisung „Pedal Trompete 8 Fuß“ für „Gottes Sohn ist kommen“ BWV 600 zu widersprechen. Diese Registrierung schließt jedoch nicht die Möglichkeit aus, im Pedal Prinzipal $8^{\prime}$ mit Trompete zu ziehen. Zudem scheint Agricola sich in erster Linie auf Manualrohrwerke zu beziehen, während Bach eine Pedaltrompete verlangt, die normalerweise von weiterer Mensur und kräftigerem, grundtönigem Klang ist. Kauffmann scheint im allgemeinen mit Agricola übereinzustimmen, ohne in dieser Frage dogmatisch zu sein.

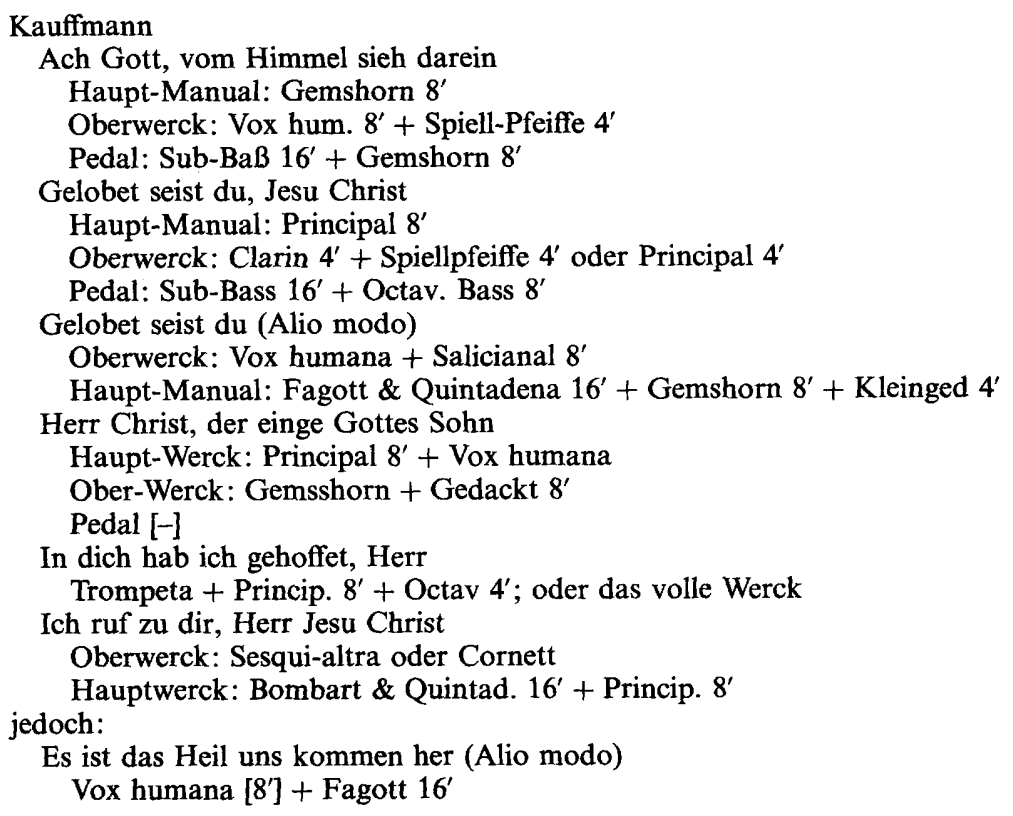

Eine der Choralvorspielregistrierungen für die Schloßkirchenorgel zu Lahm verlangt folgende Kombination im Hauptwerk:

Trompete $8^{\prime}+$ Principal $8^{\prime}$

Gronau scheint ebenfalls mit Agricola übereinzustimmen. Er setzt das Manualrohrwerk in vier Fällen ein, davon dreimal mit Beteiligung der Vox humana (stets kombiniert mit zwei oder drei Labialen). Im vierten Fall kombiniert 
Gronau eine Zungenstimme mit einer „Lücken“-Registrierung, was bei ihm einmalig ist.

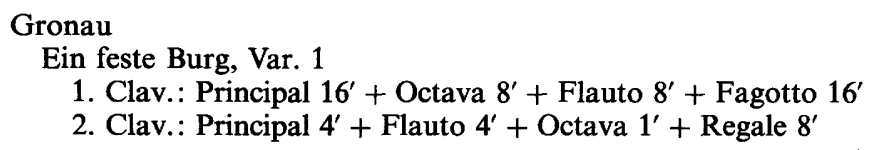

Agricola schätzt eindeutig einen runden Rohrwerkklang. Gelegentlich ${ }^{45}$ plädiert er - wie Mattheson - für das Überziehen der Pfeifenmundstücke mit Leder, um das Schnarren zu beheben. Diese ,gefütterten“ Mundstücke gewannen im Laufe des 18. Jahrhunderts an Beliebtheit. Für die Pedalposaune war das, Verfahren durchaus üblich, ${ }^{46}$ bei der Konstruktion von Manualrohrwerken jedoch folgten die Orgelbauer unterschiedlichen Vorstellungen. Gottfried Silbermann zum Beispiel fütterte die Trompetenregister des Manuals nur im $\mathrm{BaB}^{47}$ (seine Zungenstimmen entwickeln in der Regel mehr Glanz als normale mitteldeutsche Zungenregister). Adlung urteilt in dieser Frage nicht eindeutig ${ }^{48}$ und Agricola, der hier vielleicht stillschweigend die Berechtigung einer abweichenden Meinung anerkennt, enthält sich jeden Kommentars oder redaktionellen Eingriffs.

\section{Cornett-Registrierungen}

Die von Johann Sebastian Bach geschätzte Cornett-Kombination (üblicherweise Flöten $\left.8^{\prime}+4^{\prime}+22 / 3^{\prime}+2^{\prime}+13 / 5^{\prime}\right),{ }^{49}$ spielt auch in Agricolas Anleitung eine wichtige Rolle:

„Doch kann ein 8 füßig Gedackt zur Sequialtera mit der 1 füßigen Octave, bey geschwinden Brechungen, gezogen werden.

Eine Quinte oder Terze muß allezeit eine noch höhere Octave oder Superoctave über sich haben. Z. E. ist die Quinte von $3 \mathrm{FuB}$, so muß nebst dem 8 und 4 füßigen Register auch noch eine 2 füßige Stimme gezogen werden" (S. 503).

Bei der Forderung, die Aliquotstimmen müßten stets von Oktaven gedeckt sein, scheint es sich um eine Eigenart Agricolas zu handeln, der er an anderer Stelle in seinem Essay allerdings selbst widerspricht. Dort (S. 492) erläutert er die Zusammensetzung einer sechsfachen Mixtur, in der bei dem kleinen $\mathrm{c}$ der

${ }^{45}$ S. 489.

${ }^{46}$ Vgl. Adlung, Bd. 1, S. 122, § 176.

47 Vgl. Flade, a. a. O., S. 190.

${ }^{48}$ In $\S 104$ behauptet er, die Mundstücke seien ,zuweilen mit Leder überzogen, damit es nicht allzusehr knastere oder rassele; doch in manchen Registern, sonderlich in kleinen, ist dieses nicht“. In $\S 202$ heißt es über die Trompete, „die Mundstücke sind nicht gefüttert".

49 Vgl. den Umbauvorschlag für die Orgel der Mühlhäuser Blasiuskirche von 1707 (Dok I, Nr. 83). Die Orgel besaß bereits Sesquialteraregister im Hauptwerk und im Rückpositiv (vgl. Adlung, Bd. 1, S. 260 f.), doch Bach verlangte, daß diesen ein drittes Manual, ein „Brust positivgen“, hinzugefügt werden solle, von dessen sieben Stimmen fünf ein drittes Cornett bilden könnten. Über die Terz schreibt Bach, man könne „durch zuziehung einiger anderer Stimmen eine vollkommen schöne Sesquialteram [d. h. ein Cornett] zu wege bringen.“ 
höchste erklingende Ton die Terz ist. Ferner beschreibt er in seinem Bericht über die französische Registrierpraxis ein Cornett mit 13/5' als höchster Lage. Silbermann, Kauffmann und Gronau führen jeder Kombinationen mit einer Terz als höchster Lage an. Obwohl nicht völlig ausgeschlossen werden kann, daß Bach den dogmatischen Ansatz Agricolas akzeptiert hätte, ist dies doch eher unwahrscheinlich; beispielsweise hätte Bach dann das von ihm selbst vorgeschlagene Cornett für das neue Brustpositiv in Mühlhausen nicht benutzen können, ohne zugleich die dreifache Mixtur zu ziehen.

\section{Französischer Einfluß}

Agricolas Interesse an französischen Orgeln zeigt sich nicht nur in seinen Ausführungen zur französischen Registrierpraxis, sondern auch andernorts in seinem Essay ${ }^{50}$ sowie in anderen Veröffentlichungen. ${ }^{51}$ Zum Teil mag er seine Kenntnis der französischen Orgelpraxis Marpurg verdanken, der mit französischen Orgeln und Organisten wohlvertraut war und der einen deutlich französisch beeinflußten Artikel über freie Orgelimprovisation und Orgelregistrierung veröffentlicht hatte. ${ }^{52}$ Dieses Interesse entsprach der allgemeinen Vorliebe für französische Kultur, die sich in Deutschland seit dem Beginn des Jahrhunderts immer mehr durchsetzte und zu einem gewissen Grade auch von Bach geteilt wurde. ${ }^{53}$ Bach pflegte auch die Aufführung französischer Musik, ${ }^{54}$ zweifellos als Folge seiner persönlichen Eindrücke vom Spiel Marchands und anderer französischer Clavecinisten.

Der größte Hinderungsgrund für Bach (und andere deutsche Organisten), ein tieferes Verständnis für französische Registrierpraxis zu entwickeln oder diese praktisch zu nutzen, lag in den ihnen zur Verfügung stehenden Orgeln. Die Gepflogenheiten des mitteldeutschen Orgelbaus waren im 18. Jahrhundert zwar durchaus nicht einheitlich, doch alle Instrumente unterschieden sich grundsätzlich von den französischen. ${ }^{55}$ Die meisten deutschen Organisten waren mit

${ }^{50}$ Z. B. verzeichnet Agricola in einer Tabelle deutsche und französische Registerbezeichnungen (S. 501).

${ }^{51}$ Z. B. fügt Agricola dem ersten Band seiner Ausgabe von Adlungs Musica mechanica organoedi (S. 287-291) die Übersetzung eines detaillierten Berichts von Dom Bedos de Celles über eine neue Orgel in Tours bei.

52 Der Kritische Musicus an der Spree, 37. und 38. Stück, Berlin 1749 (11. und 18. November). Eine Würdigung dieses Artikels bei G. F. Crowell, Friedrich Wilhelm Marpurg (1718-1795) and French Registration Practices in Central Germany in the Middle of the Eighteenth Century, Dissertation, University of Cincinnati 1993.

${ }^{53}$ Unter den zahlreichen Belegen für französische Einflüsse ist Bachs eigenhändige Kopie von De Grignys Livre d' orgue zusammen mit seiner Abschrift von sechs Suiten François Dieuparts und der Verzierungstabelle aus D'Angleberts 1689 erschienenen Pièces de claveçin (Stadt- und Universitätsbibliothek Frankfurt/M., Mus. ms. 1538) besonders bemerkenswert.

54 Adlung berichtet, er habe Bach Suiten von Marchand spielen hören. Vgl. Anleitung zu der musicalischen Gelahrtheit, Erfurt 1758, S. 716 (Dok III, Nr. 696).

${ }^{55}$ Gottfried Silbermanns Orgeln bilden hier eine gewisse Ausnahme (denn Silbermann ging bei seinem Bruder Andreas im Elsaß in die Lehre), doch selbst sie unterscheiden sich nicht unwesentlich von zeitgenössischen französischen Instrumenten; im übrigen 
französischen Instrumenten nicht vertraut. ${ }^{56}$ Selbst Agricola, dem die französischen Gepflogenheiten noch am ehesten geläufig waren, scheint seine Kenntnisse mehr aus der Lektüre als aus dem direkten Umgang mit französischen Orgeln gewonnen zu haben. ${ }^{57}$ Die Naumburger Hildebrandt-Orgel - das einzige große Instrument, bei dessen Planung Bach beteiligt gewesen sein könnte - zeigt keine eindeutig französischen Einflüsse.

Bei der Beurteilung von Agricolas (oder Bachs) Interesse an französischen Registrierungen ist es daher notwendig, die in Mitteldeutschland herrschenden Bedingungen zu berücksichtigen: andersartige Orgeln sowie andersartige beziehungsweise anders gebrauchte Musik - prostestantisch statt katholisch und auf Chorälen statt auf Gregorianik gegründet. Im Gegensatz zu der kodifizierten und stilisierten französischen Registrierpraxis scheint Agricola (wie auch andere deutsche Autoren) weniger auf ein festes System zu zielen; beim Registrieren kann der Organist eine französisch geprägte Kombination als lediglich eine von vielen Möglichkeiten wählen - eine Auffassung, die nach Forkel auch typisch für Johann Sebastian Bach war. ${ }^{58}$

\section{Gleichzeitiger Gebrauch von verschiedenen Achtfuß-Registern}

Adlung ( $\$ 232-233$ ) verteidigt vehement die Praxis des gleichzeitigen Gebrauchs mehrerer 8'-Register auf ein und demselben Manual. Damit wendet er sich gegen die Vorschläge älterer Autoren wie Niedt und Werckmeister, die gegenteilige Meinungen vertreten ${ }^{59}$ Agricola vertritt am Schluß seiner Anmerkungen zu Fragen der Registrierung eine Position, die exakt derjenigen Adlungs entspricht. Auch Kauffmann und Gronau befürworteten diese Praxis:

Kauffmann

Es ist das Heil

Gedact + Spitzflöth + Viola [8']; item das volle Werck

Vater unser im Himmelreich (Alio modo)

Rückposit: Quintad $8^{\prime}+$ Flaut douc $8^{\prime}$

Oberwerck: Vox hum $8^{\prime}+$ Gemsshorn $8^{\prime}$

Gronau

Was Gott thut das ist wohlgethan, Var. 2

Man.: Principal $8^{\prime}+$ Flauto $8^{\prime}+$ Octava $4^{\prime}+$ Salicetto $4^{\prime}$

Ped.: Principal $16^{\prime}+$ Basso Coperto $16^{\prime}+$ Violone $16^{\prime}+$ Octava $8^{\prime}+$ Cornetto $4^{\prime}$

fanden Bach und Agricola (wie oben erwähnt) die Orgeln Silbermanns in einer Reihe von Aspekten alles andere als ideal.

56 Vgl. Adlung, Bd. 1, S. 287, wo Agricola bestätigt, daß französische Orgeln wenig bekannt waren.

57 Auf S. 505 seines Essays gibt Agricola dies fast zu.

58 Vgl. Forkel, S. 21: „Solche Beobachtungen [über Akustik] konnten und mußten ihn allerdings auch auf Versuche führen, durch ungewöhnliche Vereinigung verschiedener Orgelstimmen vor und nach ihm unbekannte Wirkungen hervor zu bringen."

59 F. E. Niedt, Musicalischer Handleitung anderer Teil |Von der Variation Des GeneralBasses ... Zweite Auflage ... mit ... einem Anhang von mehr als 60. Orgel-Wercken, versehen durch J. Mattheson, Hamburg 1721, S. 116; A. Werckmeister, Erweiterte und verbesserte Orgel-Probe, Quedlinburg 1698, S. 72. 
Das wichtigste Merkmal von Gronaus Registrierungspraxis ist praktisch eine Ballung von $8^{\prime}$ - und 4'-Klängen, die gelegentlich durch eine Flöte $2^{\prime}$ oder Superoktave belebt werden. Lediglich Silbermann hält kritische Distanz zu vielfachen Äqualstimmen. Für das Plenum schlägt er zwar Prinzipale und Flöten vor, doch im übrigen bevorzugt er Kombinationen von Registern in verschiedenen Lagen.

Agricola beschließt seine Abhandlung mit einem besonders bemerkenswerten Hinweis:

„Ich habe in einer gewissen Orgel das liebliche Gedackt, die Vugara, die Quintadene und die Hohlflöte alle von $8 \mathrm{FuB}$, ohne irgend eine andere Stimme, zusammen gehöret, welches eine schöne und fremde Wirkung that" (S. 505).

Da diese vier Register in mitteldeutschen Orgeln des 18. Jahrhunderts außerordentlich selten auf einem einzigen Manual auftreten, besteht kaum ein Zweifel, da $B$ jene „gewisse Orgel“ keine andere ist als das von Gottfried Heinrich Trost für die Altenburger Schloßkirche erbaute Instrument. Die vier genannten Stimmen finden sich hier im Oberwerk, und zwar in nahezu gleichlautender Schreibweise wie bei Agricola.$^{60}$ Altenburger Dokumente belegen, daß Bach das Instrument kurz nach dessen Fertigstellung im September 1739 (zur Zeit von Agricolas Studienaufenthalt in Leipzig) spielte und es für gelungen befand. ${ }^{61}$ Diese bemerkenswerten Übereinstimmungen rufen zum einen die Passage des Nekrologs zu Bachs Registrierkunst ins Gedächtnis:

„Er verstund ... die Art die Orgeln zu handhaben, die Stimmen derselben auf das geschickteste mit einander zu vereinigen ... in der größten Vollkommenheit" (Dok III, Nr. 666, S. 88).

Zum anderen erinnern sie an Forkels anekdotischen Bericht über Bachs aufsehenerregende Registerkombinationen. ${ }^{62}$ Sollte es sich bei dem von Agricola beschriebenen Erlebnis etwa um Bachs Orgelspiel in Altenburg gehandelt haben? Erinnerte sich Agricola, als er den Nekrolog verfaßte, vielleicht unter anderem an die Altenburger Orgelerprobung im September 1739? Und ist vielleicht auch Forkels Bericht über Bachs Registrierkunst unter Umständen ein fernes Echo einer solchen Begebenheit?

Obwohl die erreichbaren Quellen zu Registrierungsfragen vielfach keine schlüssigen Antworten liefern, bieten sie doch Informationen, die alles andere als dürftig sind. Vielmehr vermitteln sie einen reichen Wissensfundus ergiebig genug, um weitreichende Übereinstimmungen wie auch offensichtliche und verborgene Widersprüche aufzuzeigen, wie sie sich in jedem Zeitalter und in jedem Fach als unvermeidliche Begleiterscheinung subjektiver Präferenzen ergeben. Agricolas Abhandlung gestattet nicht nur eine große Annäherung an Bachs Konzeption der Registrierpraxis, sondern vermittelt auch

${ }^{60} \mathrm{Vgl}$. die von Agricola in Adlung, Bd. 1, S. 286, mitgeteilte Disposition.

${ }^{61}$ Vgl. Dok II, Nr. 453.

${ }^{62}$ Forkel, S. 20. 
einen verhältnismäßig umfassenden Überblick. Es erscheint daher sinnvoll, aus der Fülle der Detailinformationen, die sich aus dem vorstehend durchgeführten Quellenvergleich ergeben, die folgenden thesenartigen Schlußfolgerungen zu ziehen und damit die Bedeutung von Agricolas Arbeit in das gebührende Licht zu rücken.

1. Agricolas Feststellungen können nicht aufgrund der Tatsache verworfen werden, daß ihr Autor nicht der Generation Bachs, sondern der Folgegeneration angehörte. Seine Abhandlung offenbart weitreichende Übereinstimmungen mit Äußerungen von Georg Friedrich Kauffmann (1679-1735), Johann Friedrich Walther (um 1727), Jacob Adlung (1699-1762) und sogar Daniel Magnus Gronau (ca. 1700-1747) im fernen Danzig. Sie alle waren Zeitgenossen Bachs.

2. Alternative Registrierungen gegenüber dem Plenum sind - wie Kauffmann bezeugt - vorstellbar. Agricola verdeutlicht, $\mathrm{da} ß \mathrm{C}_{\mathrm{j}}$ die eigentliche Vielfalt der Registrierungskunst nicht in den Vorschriften für das Plenum, sondern in der Kombination von Flötenstimmen und Streicherstimmen (von Agricola als „Galanteriestimmen “63 bezeichnet) und im Gebrauch von Rohrwerken in Verbindung mit Labialen besteht.

3. Die auf dem Umweg über Agricola tradierte Registrierpraxis Bachs erscheint weder avantgardistisch noch völlig konventionell. In manchen Aspekten, so in der gleichzeitigen Verwendung verschiedenartiger Achtfuß-Register, stimmt Agricola mit progressiven Orgelspezialisten seiner Zeit (beispielsweise Adlung) überein, in anderen (etwa der Beschränkung des Plenums auf den Prinzipalchor) wirkt er verhältnismäßig konservativ.

4. Die Registrierpraxis des 18. Jahrhunderts scheint abwechslungsreicher und kühner gewesen zu sein, als heutige historisch orientierte Musiker zuzugeben bereit sind beziehungsweise neuzeitlicher Geschmack zu tolerieren gewillt ist. Adlungs freizügige Handhabung von Registrierungen, das häufige Vorkommen eigenwilliger Kombinationen (etwa $16^{\prime}+4^{\prime}, 16^{\prime}+2^{\prime}$ und $16^{\prime}+1^{\prime}$ ) sowie kompakte $16^{\prime}+8^{\prime}$-Klänge, schließlich die Existenz von Orgeln mit vielen verschiedenfarbigen $8^{\prime}$-Registern (einschließlich Streicherstimmen) sowie Effektregistern wie Glockenspiel oder Vox humana (ein sanft schwebendes Prinzipalregister) - all dies bezeugt eine ungezwungene und phantasievolle Registrierpraxis.

5. Die in Agricolas Essay verstreut auftretenden Bemerkungen zu Orgeln und Orgelbauern (etwa Altenburg und Christian Ernst Friderici) bestätigen die gegenseitige Beeinflussung von Orgelbau und Orgelspiel einschließlich der Registrierung. Praktische Erfahrung auf dem Instrumententyp, mit dem Bach und Agricola vertraut waren, liefert weit wertvollere Erkenntnisse zur

${ }^{63}$ S. 499. 
Registrierpraxis als jede schriftliche Quelle, ergänzt und vertieft überdies die aus den Quellen gewonnene Information. Jede Diskussion von Bachs Registrierkunst muß daher diese Orgeln berücksichtigen, von denen einige in unterschiedlichen Restaurierungs-Stadien erhalten sind.

Den veröffentlichten Dispositionen der vielen sogenannten Bach-Orgeln, ${ }^{64}$ auch der von Bach über längere Zeit gespielten Instrumente, sollte mit Vorsicht begegnet werden, da viele von diesen nicht mehr vorhanden sind (darunter vor allem diejenigen in Arnstadt und Weimar, für die Bach den größten Teil seines Orgelwerks geschaffen hat) und somit keine genauere Vorstellung von deren Klang $\mathrm{zu}$ gewinnen ist. Ferner ist unbekannt, in welchem Maße die Bach zur Verfügung stehenden Instrumente auch wirklich seinen Klangvorstellungen entsprachen. ${ }^{65}$ Unter den erhaltenen, nachweislich von Bach gespielten Orgeln ist das einzige bedeutende, annähernd im Originalzustand befindliche Instrument die Orgel der Schloßkirche zu Altenburg, von der wir zudem wissen, daß er ihren Klang schätzte. ${ }^{66}$ Glücklicherweise ist dieses Instrument ein überragendes Beispiel für jenen eigentümlichen thüringischen Orgeltyp des späten 17. und frühen 18. Jahrhunderts, ${ }^{67}$ mit dem Bach am engsten vertraut war. Es gibt noch weitere Orgeln dieses Typs - manche in schlechtem Erhaltungszustand -, darunter Instrumente von Sterzing, Wender, Volkland, Trost, den Hildebrandts (Vater und Sohn) und Friderici, jedoch ist seit dem 18. Jahrhundert kein derartiges Instrument mehr gebaut worden und kein einziges existiert außerhalb Mitteldeutschlands. Richtig zu verstehen sind die von Agricola diskutierten Registrierungsmöglichkeiten einzig im Kontext der Instrumente, für die sie ursprünglich gedacht waren.

\section{ANHANG}

Zur inhaltlichen Ergänzung des vorstehenden Aufsatzes wird nachfolgend ein von Johann Friedrich Agricola verfaßter und geschriebener Text für eine

${ }^{64}$ Vgl. z. B. W. Schrammek, Bach-Orgeln in Thüringen und Sachsen, in: Johann Sebastian Bach. Lebendiges Erbe, Leipzig 1983 (Beiträge zur Bachpflege. 11.); H. D. Blanchard, The Bach Organ Book, Delaware (Ohio) 1985.

${ }^{65}$ Vgl. den Nekrolog: „Aller dieser Orgelwissenschaft ungeachtet, hat es ihm, wie er oftmals zu bedauren pflegte, doch nie so gut werden können, eine recht grosse und recht schöne Orgel zu seinem beständigen Gebrauche gegenwärtig zu haben“ (Dok III, Nr. 666, S. 88).

${ }^{66}$ Ein weiteres erhaltenes Instrument, potentiell vielleicht noch bedeutender als Altenburg, ist die Hildebrandt-Orgel der Wenzelskirche zu Naumburg (Bach war mit Hildebrandt befreundet und wirkte möglicherweise am Entwurf dieses Instruments mit; vgl. Dok I, Nr. 90 und Nr. 88). In ihrem Gutachten (1746) bringen Bach und Silbermann ihre Zufriedenheit mit dem Instrument zum Ausdruck (Dok I, Nr. 90); auch Agricola rühmt es uneingeschränkt (vgl. Adlung, Bd. 1, Anmerkung zu § 324). Diese Orgel ist jedoch durch spätere Reparaturen und Umbauten in ihrem Charakter so grundlegend verändert worden, daß ihre wahre Bedeutung erst nach einfühlender Wiederherstellung durch einen engagierten Restaurator eingeschätzt werden kann.

${ }^{67}$ Für eine eingehende Diskussion der Merkmale dieser Orgeln siehe L. Edwards, The Thuringian Organ 1702-1720, in: The Organ Yearbook 22, 1991, S. 119-150. 
Orgelprobe erstmals abgedruckt.* Er bietet einen Beleg für die organistische Autorität, die Agricola in Berlin zugebilligt wurde.

„Foderungen an den Candidaten des Organistendienstes bey der Kirche zu St. Nicolai in Berlin, die ihm eine Viertelstunde vor der Probe vorgelegt worden.

Der Herr Candidat wird bey der itzo eben abzulegenden Probe belieben:

1) Aus freyer Erfindung mit dem vollen Werke zu präludiren; und zwar im $\mathrm{H}$ dur anzufangen, und im D moll aufzuhören.

2) Ein besonderes Vorspiel auf den Choral: Christ unser Herr zum Jordan kam, auch aus freyer Erfindung, zu machen. Der Cantus firmus oder eigentliche Gesang dieses Chorals muß dabey angebracht, und auf einem stärker angezogenen Claviere vorgetragen werden. Die dazu, nach der Erfindung des Spielers, modulirenden Stimmen werden auf einem andern schwächer angezogenen Claviere vorgetragen. Das Pedal ist dabey nicht $\mathrm{zu}$ vergessen.

3) Eben diesen Choral: Christ unser Herr etc. etc. ganz simpel, aber vollstimmig, so wie er zum Gesange der Gemeine gespielet werden muß, vorzutragen. Ein Vers davon versteht sich.

4) Eine Sing=Arie, oder ein ganz Kirchenstück, welches der Hr. Cantor Ditmar dazu hergeben wird, mit dem Generalbasse zu begleiten.

5) Zum Beschlusse entweder ein Orgelstück eines guten Meisters, welches er sich wählen kann, nach Noten, oder, nach Belieben, eine freye Fantasie aus eigenem Kopfe, hören zu lassen: im letztern Fall aber, dabey mit den 3 different angezogenen Clavieren geschickt abzuwechseln.

Berlin am 1. Februar, 1773

Johann Friedrich Agricola“

* Landesarchiv Berlin, Rep. 04-02-1, Kirchenabteilung des Magistrats Nr. 578 (Organisten der Nikolai-Kirche, 1681-1917). Zum Sachverhalt vgl. Curt Sachs, Musikgeschichte der Stadt Berlin bis zum Jahre 1800, Berlin 1908, S. 115. Die Redaktion dankt Dr. Peter Wollny (Leipzig) für den Hinweis auf diese Quelle. 